\title{
Clinical Challenges in Managing Minor Developmental Anomalies in Children
}

\author{
Kanu Priya Gupta1, Partapjot S. Grewal'², Shalini Garg ${ }^{3}$
}

\begin{abstract}
The close relationship among oral, systemic and psychologic health requires that oral health be evaluated thoroughly as part of health maintenance supervision. An understanding of normal sequence and patterns of tooth eruption is the foundation for identifying and treating children with abnormal dental development. Distinguishing normal from pathological dental development requires careful evaluation of the patient, including medical, dental and family history, clinical examination, radiographic evaluation and possibly special laboratory tests.Developmental anomalies of teeth are marked deviations from the normal standards in color, contour, size, number, and degree of development of the teeth. Systemic as well as local factors may operate to produce these developmental disturbances. These developmental anomalies have multiple, complex problems as they affect both form and function and can have significant psychological impact. These conditions may present early in life and require both immediate intervention and management of a protracted nature, including coordination of multi-disciplinary carealongwith accurate diagnosis, timely intervention with a comprehensive treatment plan. This paper presents a clinical update on the various dental anomalies alongwith their management options and consequences.
\end{abstract}

Keywords: Dental anomalies, young age, oral rehabilitation, time for intervention

${ }^{1}$ Senior Lecturer,

Deptt of Pedodontics \& Preventive Dentistry, National Dental College \& Hospital,

Derabassi, Punjab, India

${ }^{2}$ Senior Lecturer,

Deptt of Prosthodontics,

BRS Dental College,

Barwala, Haryana, India

${ }^{3}$ Proff \& HOD,

Deptt of Pedodontics \& Preventive Dentistry,

M.M. Dental College,

Mullana, Ambala, India

\author{
Contact Author \\ Dr. Kanu Priya Gupta \\ dentico_kanupriya@yahoo.com \\ J Oral Health Comm Dent 2011;5(3)136-141
}

\section{INTRODUCTION}

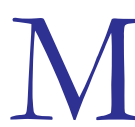

inor developmental anomalies are mild malformations with primarily morphological involvement. They represent a morphological defect, an irreversible sequel of disturbed morphogenesis. Anomalies of the oral and dental structures can be isolated or may be a symptom of a major defect or manifestation of a general disorder or syndrome. The recognition and identification of orodentalminor anomalies are of great importance for timely and accurate diagnosis of numerous genetic abnormalities of the craniofacial region. Marden et al defined minor anomalies as structural anomalies without medical and genetic sequels, and with prevalence below 4\% in the general population. $\mathrm{R}$ Mehes et al consider that minor anomalies develop consequentially to morphogenesis disturbances, and are frequently associated with congenital disturbances in wide terms (1).

A variety of dental anomalies are associated with defects in tooth development related to hereditary, systemic, traumatic, or local factors. Dental anomalies are variations in the number, size, and shape of teeth. In addition, a disruption during tooth development may affect the structure or the color of the teeth. Besides, these anomalies can also affect the developing occlusion in young children and lead to malocclusions in children.

So, in view of all these considerations here is a guide to clinical diagnosis and management of various dental anomalies commonly seen in children during mixed dentitions as this is one of the most challenging period for a clinician when one has to plan the treatment keeping in mind various parameters for the developing child.

\section{DISTURBANCE IN TEETH SIZE Microdontia}

This refers to decrease in tooth size, can be generalized/localized microdontia affecting a single tooth. Most commonly affected teeth are the maxillary laterals, called as Peg laterals (Figure 1) followed with the third molars. A peculiar case is of microdontia involving the mandibular second premolar in a young child is shown (Figure 2a,2b). 


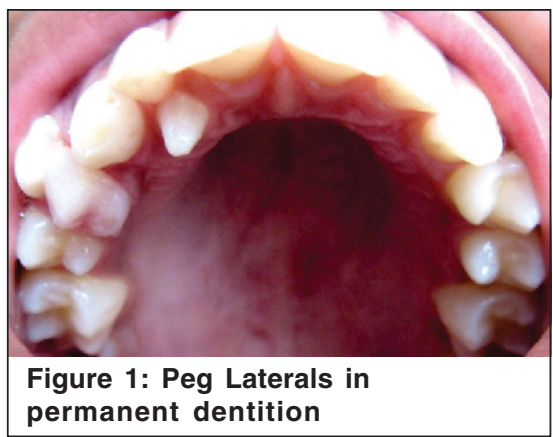

Treatment: Managing microdontia i.r.t. peg laterals involves composite/ esthetic build ups for these teeth. In case of a permanent premolar as in (Figure 2), one can go for interim restorations like acrylic crowns or stainless steel crowns in mixed dentitions (Figure 2c) until the permanent dentition is established. These can be replaced with permanent full coverage crowns like ceramic or ceramic used to metal crowns as they have to bear the masticatory forces in this area.

\section{Macrodontia: Synonyms - Megadontia, Megalodontia}

This is an abnormal large size tooth, which can be true/relative \& localized/ generalized. (Figure 3a). shows macrodont maxillary central incisors alongwiththe radiograph of the same showing incomplete root formation and wide pulp chambers (Figure 3b). They were a major concern of esthetics leading to crowding and malocclusion in this 9 year old young girl.

- Treatment: Post operative view (Figure 3c) shows restored occlusion, arch form and esthetics of the patient achieved after extracting the nonrestorable right maxillary central incisor, interceptive orthodontic intervention \&esthetic composite build up of right

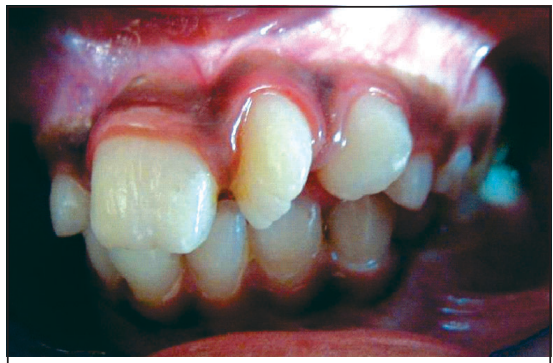

Figure 3a: Macrodont central incisors in a 9 year old child

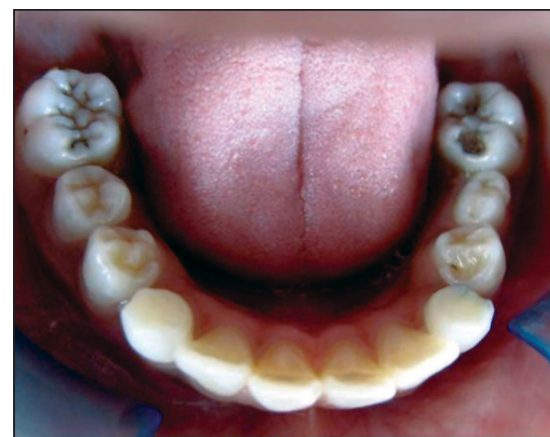

Figure 2 a: Pre-operative intra-oral view of microdontia of permanent second premolar

lateral incisor giving it the shape of a central incisor. Managing macrodonts usually involves tracing the cause of macrodontia i.e. whether it is due to the fusion with a supernumerary tooth or true enlarged tooth. One should keep in mind all the related factors and then decide for the final intervention.

\section{DISTURBANCE IN NUMBER OF TEETH}

\section{Partial anodontia}

Partial anodontia may involve a single tooth or a group of teeth. The incidence of this is less in primary dentition $(<1 \%)$ compared to the permanent dentition. Here is presented few cases with partial anodontia in primary as well as permanent dentition. Figure 4a shows a 5year old child suffering from rampant caries with partial

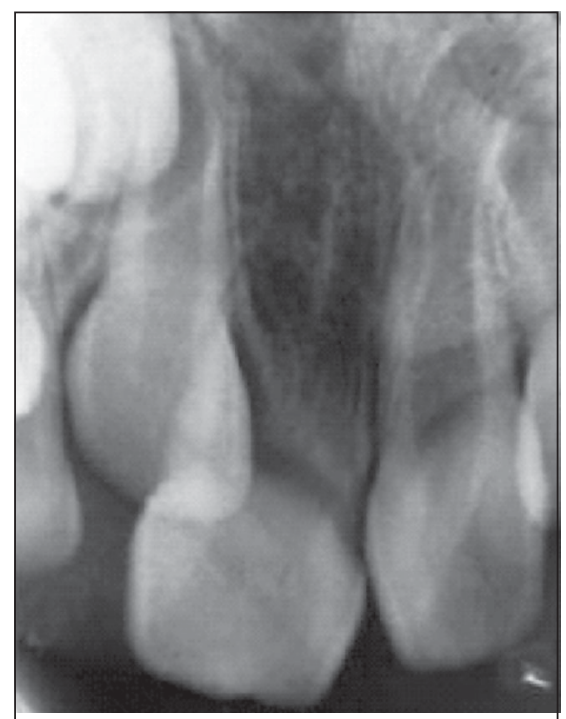

Figure $3 \mathrm{~b}$ : Intra oral radiograph of the macrodont central incisors

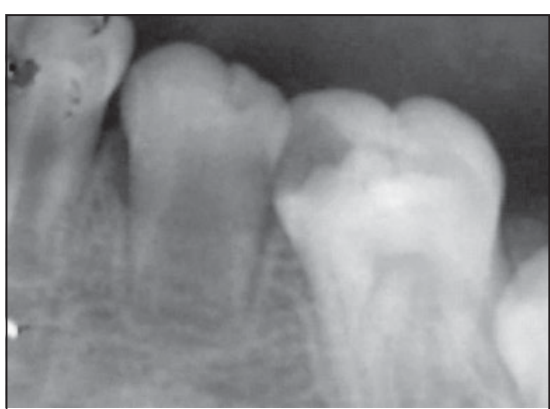

Figure 2 b: Pre-operative radiographic view of microdontia of permanent second premolar

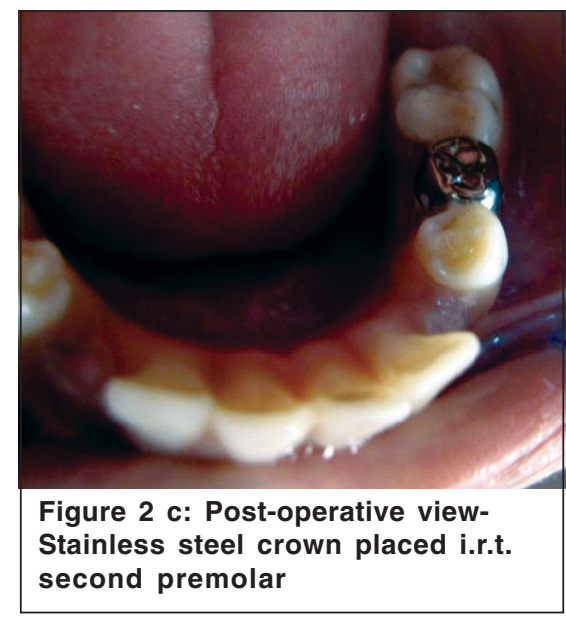

anodontia i.r.t. the primary as well as permanent mandibular central incisors (Figure 4b).

This was a rare case where the primary as well as permanent mandibular incisors were missing in a 5year old child.Another case of partial anodontia involving the permanent mandibular second premolar was observed in mixed dentition in an 11year old child (Figure 5).

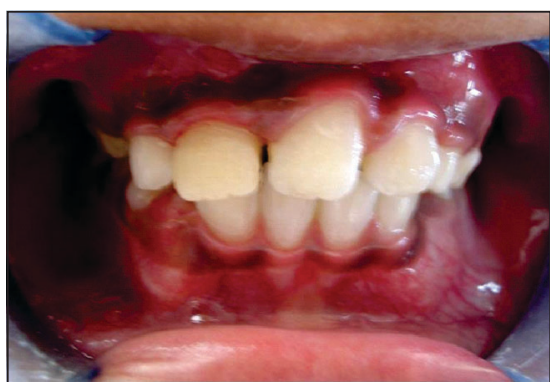

Figure 3c: Post operative view of macrodonts after orthodontic and esthetic management 

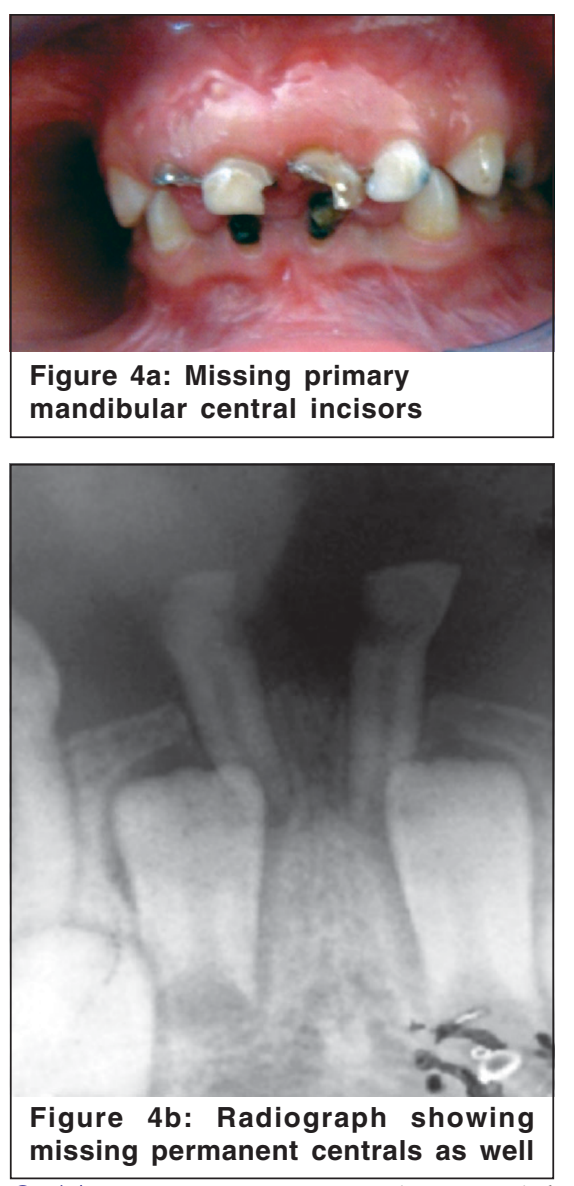

- Treatment: In managing partial anodontia, the age of the child as well as the current occlusal status with space analysis should be considered. In our 5 year old patient as no orthodontic intervention was needed (no space problem) at this deciduous time, we opted for oral rehabilitation for the rampant caries and wait and watch therapy for the permanents to erupt and occupy their respective place along with arch growth. In case of the child in his late mixed dentition,retained deciduous teeth was preserved with endodontic treatment (Obturation

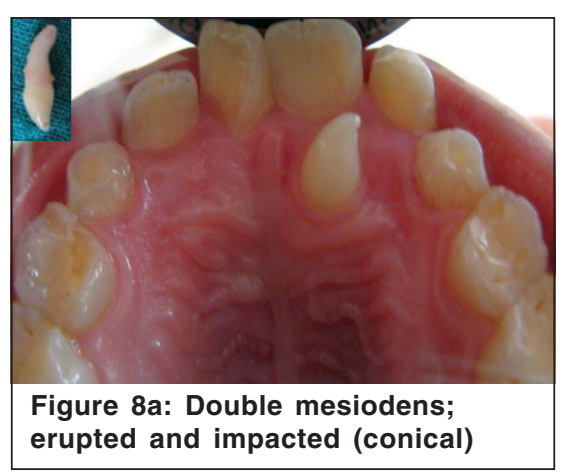

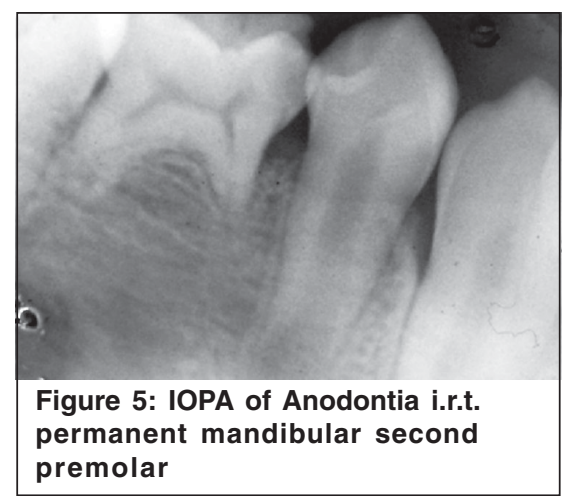

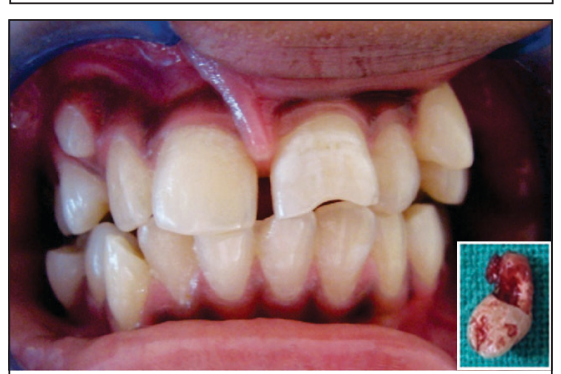

Figure 7a: High labial frenum causing midline diastema(Insetextracted mesiodens)

with Gutta percha instead of usual resorbable materials in primary teeth) and interim tooth restoration.

\section{Hyperdontia}

It is the increase in tooth number,this developmental anomaly is indicative of hyperactivity of dental lamina. An extra tooth in the oral cavity can be a mesiodens (if present in between the two maxillary central incisors), paramolar (around the last permanent molar), distomolar (if present distal to the last erupted permanent molar). According to the shape these can be conical, supplemental, tuberculate or odontomas (2). They are least prevalent in the primary

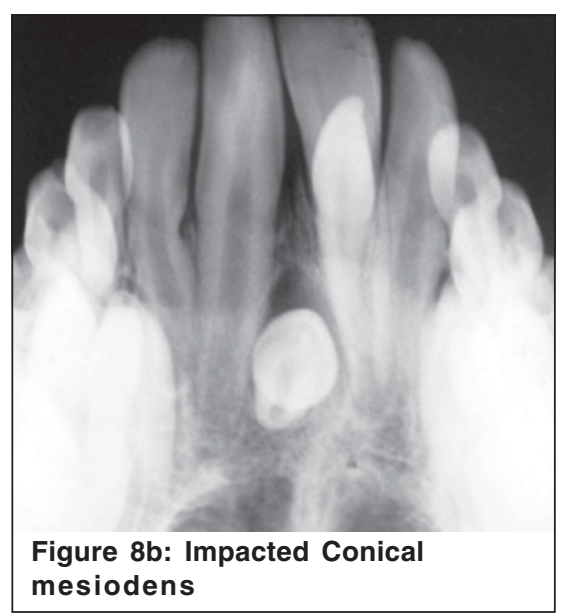

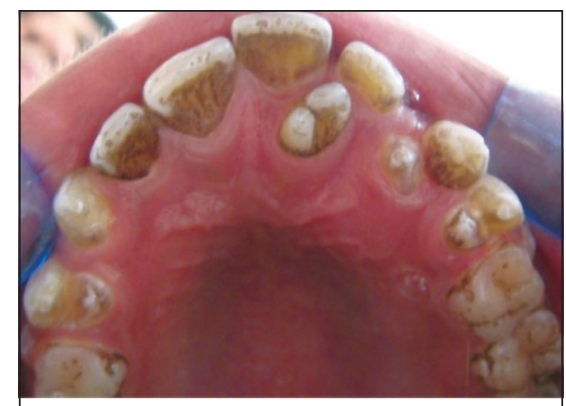

Figure 6: Tuberculatemesiodens

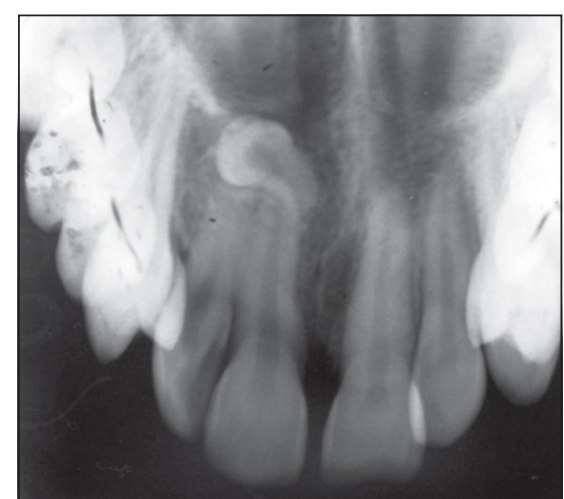

Figure 7b: Maxillary anterior occlusal radiograph showing inverted mesiodens as well

dentition (0.3-0.8\%) (3). The preferred treatment intervention for such teeth is extraction or surgical removal as these may lead to malocclusion and various eruption problems for their adjacent teeth. (Figure 6) shows an erupted mesiodens with tuberculate form that was extracted \& occlusion restored of the patient. (Figure7a) was a case of a young girl with high frenum as well as inverted mesiodens (Figure 7b) that was extracted surgically.Occlusion was restored and midline diastema was closed.

Another peculiar case was of a double mesiodens: erupted as well as impactedconical form (Figure 8a\&8b). Treatment of

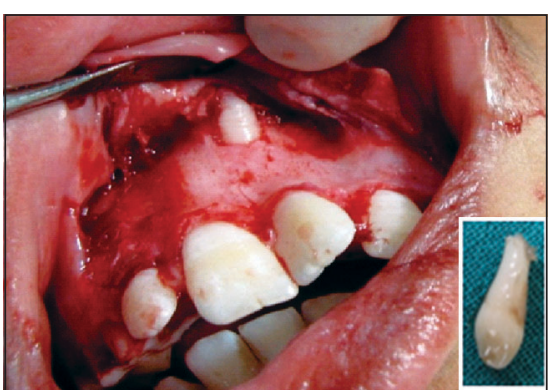

Figure 8c: Impacted mesiodens located and extracted surgically 


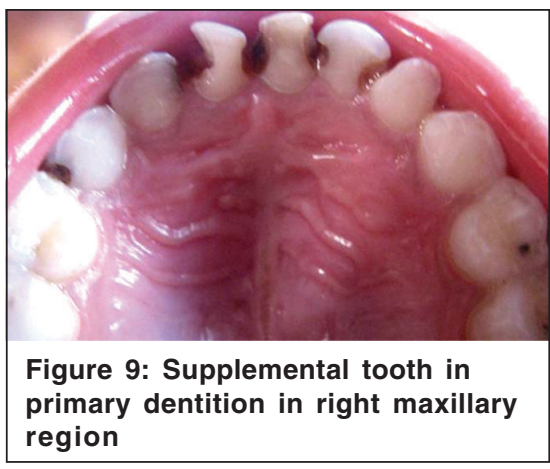

choice was extraction of the two, with the impacted one extracted surgicallyto restore the occlusion of the child (Figure 8c) (Figure 9) shows a supplemental tooth in maxillary right anterior region in a 5 year old child that is a rare occurrence.

- Treatment: Plan of treatment is to wait and watch as no malocclusion or compromised function was observed. However, esthetic and functional rehabilitation was performed for enhanced esthetics, phonetics and psychological assurance.

\section{DISTURBANCE IN STRUCTUREOF TEETH}

A disruption during tooth development may affect the structure or color of the teeth. For example, enamel hypoplasiai.e. insufficient and/or irregular quantity of enamel, may affect tooth structure, and enamel hypocalcification i.e. a condition in which the enamel is normal, but the enamel quality is poorthat may affect tooth color. (Figure 10a) shows such a case of enamel hypocalcification i.r.t. maxillary central incisor. Treatment: for such teeth is composite veneering after excavating the lesion or laminates if its an adult and tooth involvement is extensive (Figure 10b).

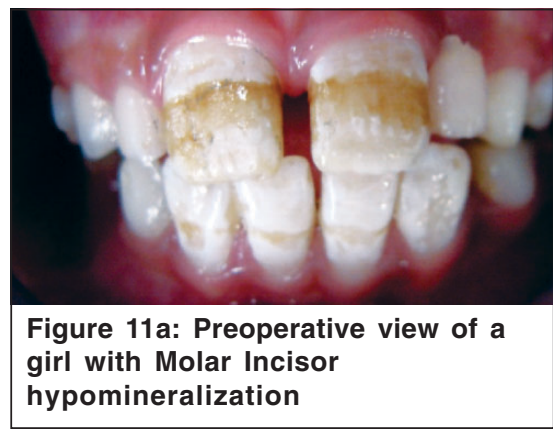

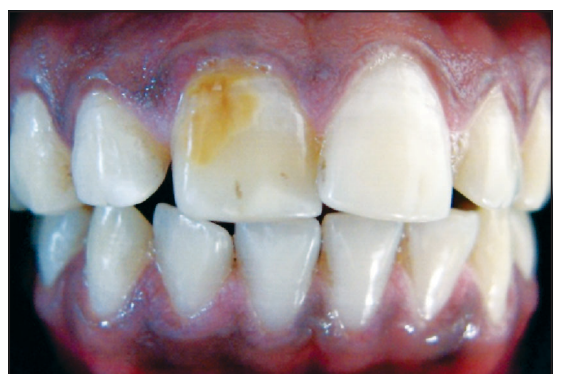

Figure 10a: Pre-operative hypocalcified ${ }^{11}$

Molar Incisor Hypo mineralization

Molar Incisor Hypo mineralization is a relatively common condition that varies in clinical severity and can result in early loss of the permanent 6-year molars. The etiology of MIH remains unclear, and the diagnosis can be confused with more generalized enamel defects such as those that occur in amelogenesis imperfecta. The management of MIH depends largely on the severity of the enamel defect. Degrees of hypomineralization can range from mild enamel opacities to enamel that readily abrades from the tooth as it emerges into the oral cavity. Usually, severely affected molars are extremely hypersensitive, prone to rapid caries development, and can be difficult to manage in young patients. Diagnostic criteria to establish the presence of MIHinclude: the presence of a demarcated opacity (defectaltering the translucency of the enamel), posteruptive enamel breakdown (loss of surface enamel after tootheruption, usually associated with a pre-existing opacity), and atypical restorations (reflecting the distribution of hypoplastic enamel). The presence of atypical restorations (Figure 12 a), especially inpatients with a relatively low caries rate, is often indicativeof MIH as the etiology

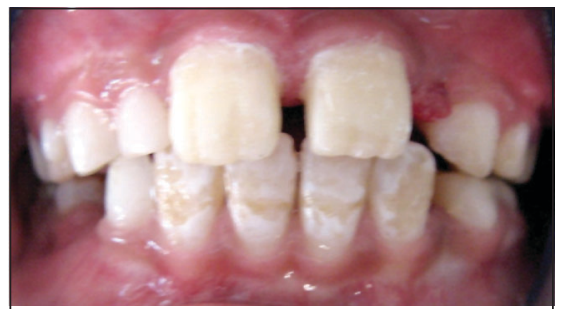

Figure 11b: Post-operative view after esthetic composite build ups as interim treatment option

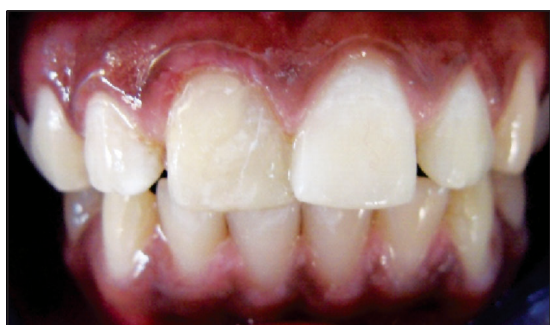

Figure $10 \mathrm{~b}$ : Post-operative view of 11 after esthetic composite veneering

leading to these type of restoration $(4,5)$.

- Treatment: of choice for such extensively involved young permanent molars is preformed stainless-steel crowns for severely hypoplastic molars (Figure 12b). Stainless-steel crowns protect the tooth againstmasticatory forces, protect enamel from acid attack andconsequent caries, decrease sensitivity and can potentiallyincrease the child's oral hygiene compliance. These act as a satisfying interim treatment option in young dentitions without compromising excessive tooth structure and restoring function as well.

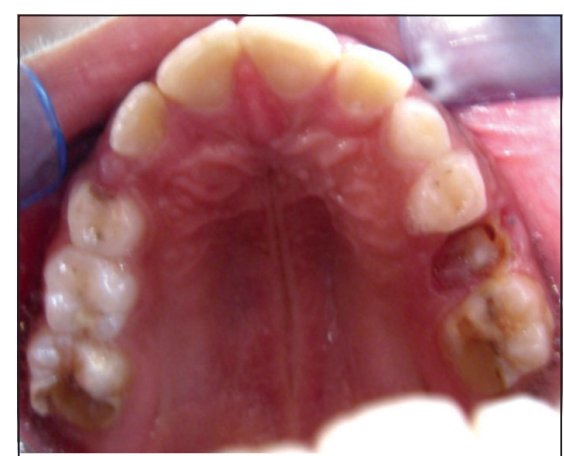

Figure 12 a: Atypical restorations in a MIH patient

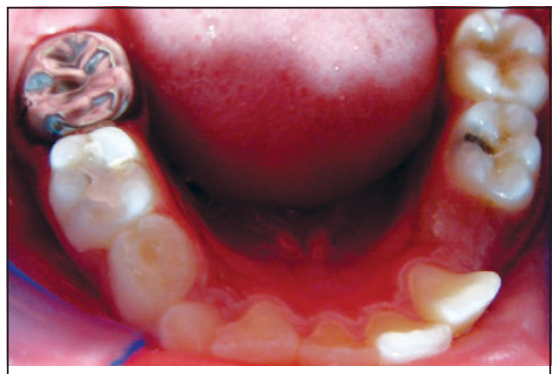

Figure 12 b: Preformed Stainless steel crowns placed in young permanent molars 


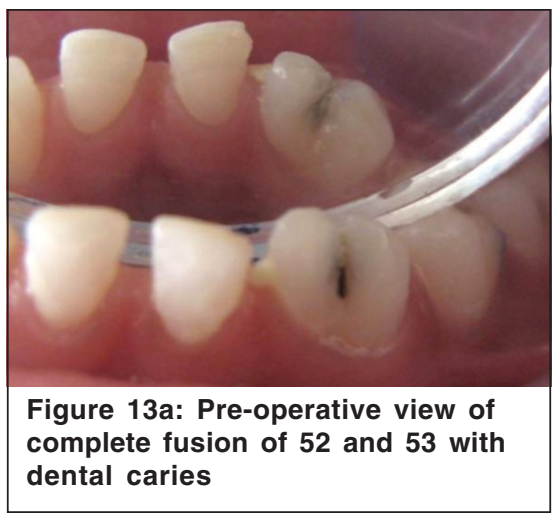

\section{DISTURBANCE IN SHAPEOF TEETH} Fusion (syndopdontia)

Fusion is one of the primary developmental anomaly where in the two teeth are fused either partially/completely through their crowns/ roots. The diagnostic criterion for this anomaly is the reduction in the tooth number in the oral cavity. In the primary dentition, the frequency of gemination or fusion is about $2.5 \%$. Bilateral presentation is very rare $(6,7)$. In the first case (Figure 13 a), it was

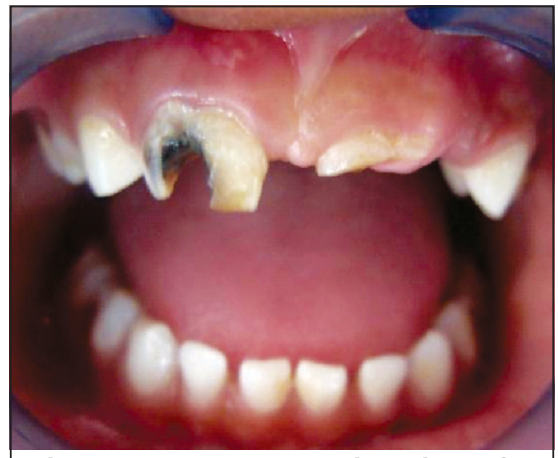

Figure 14 a: Pre-operative view of grossly carious fused $(51,52)$ and $(61,62)$

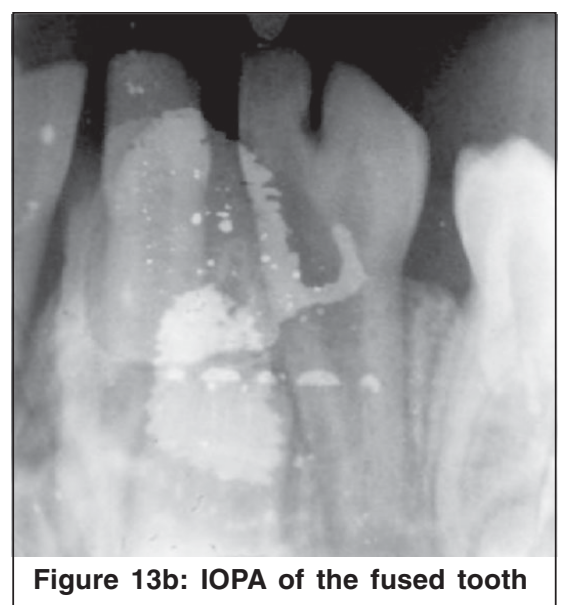

mandibular left primary lateral incisor and canine showing complete fusion of crown as well as root with a single pulp chamber. Dental caries was observed at the line of fusion. Treatment: So to prevent further involvement of the tooth, it was restored with the recent resin modified GIC as a corrective as well as preventive treatment modality as it is esthetic and has the ability to release fluoride. Thus further progress of caries was prevented and the tooth vitality restored (Figure $13 \mathrm{c}$ ).

Another case of bilateral fusion of primary maxillary central and lateral incisors is reported in a 5 year old child where a complete fusion of the crowns was observed with no involvement of roots (Figure 14 a). The extent of caries for these teeth made them non-restorable. Treatment of choice for these was extraction followed with a fixed functional space maintainer to restore esthetics, function and phonetics (Figure14 b).
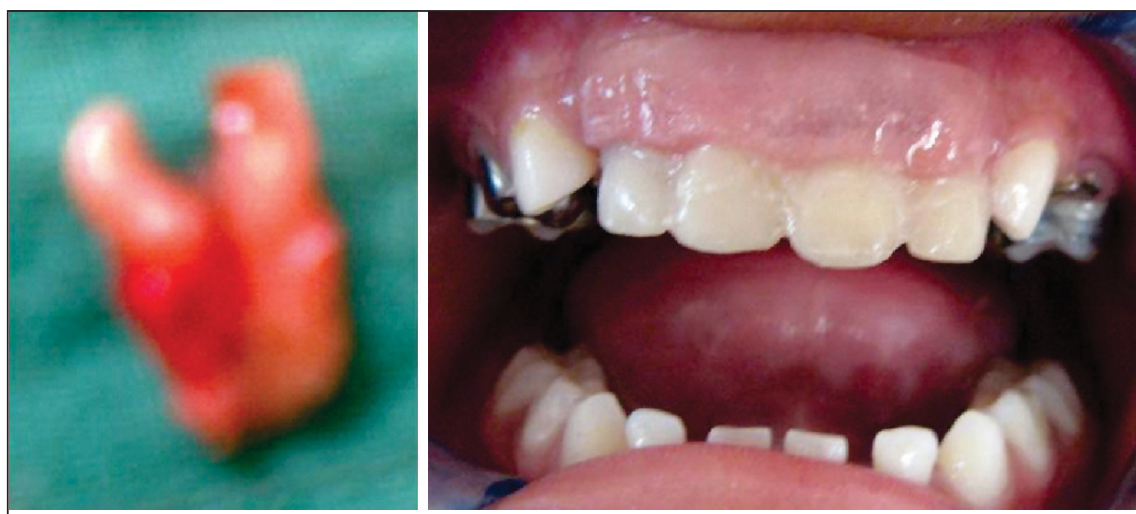

Figure 14 b: Fused teeth extracted and fixed functional prosthesis delivered

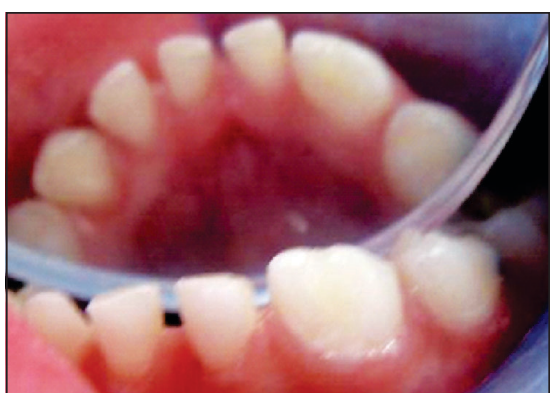

Figure 13c: Resin modified GIC i.r.t. the involved tooth

\section{Concrescence}

This is another type of fusion which occurs after the root formation hence the fused teeth are joined only by the cementum (roots). Clinically such teeth are not diagnosed easily, clinician may discover it accidentally on radiograph. Treatment includes extraction of these teeth if they are causing any malocclusion or hindering the eruption of their succedaneum tooth. Clinical significance includes difficulty in extraction or retention in case of deciduous teeth (Figure 15).

\section{Accessory / Supernumerary Cusp}

These include the cusp of Carabelli on permanent molars or deciduous molars, Talon's Cusp that projects from the lingual surface of primary or permanent anterior teeth. It is morphologically well delineated $\&$ extends at least half the distance from the cement-enamel junction to the incisal edge (Davis \& Brook). Occurs with a frequency of $0.04-10 \%$ but the etiology still remains unknown. As with other abnormalities of tooth shape and size,

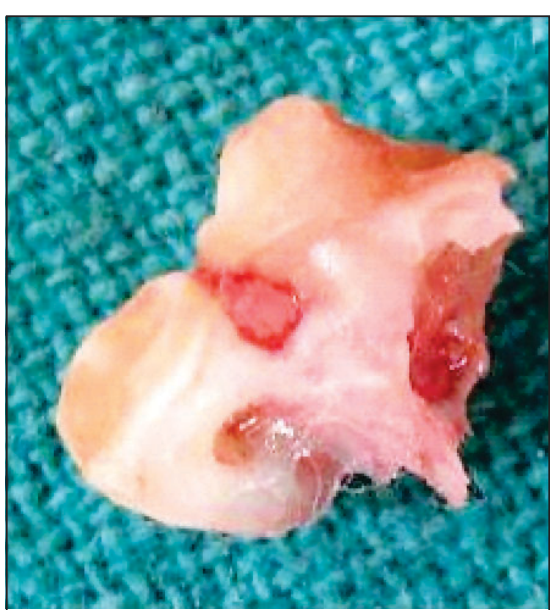

Figure 15: Concrescence IRT(73,74) 


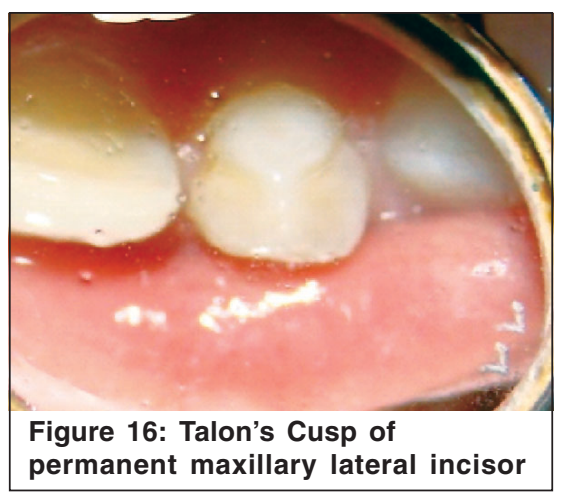

talon cusp occurs early in odontogenesis, i.e., during the morpho-differentiation stage. It may occur as a result of outward folding of the inner enamel epithelial cells (precursors of ameloblasts) and a transient focal hyperplasia of the mesenchymal dental papilla (precursors of odontoblasts) (8). Clinically these can cause malocclusion, tongue irritation or caries along the line of fusion with the teeth.

- Treatment: Because the extra tubercles have effect on the occlusal interference and can cause occlusal trauma the treatment plan called for a gradual reduction of the talon cusps on consecutive visits and an application of fluoride at each visit. Endodontic treatment is accomplished if pulpal involvement is there.

\section{SUMMARY AND CONCLUSION}

To conclude, this article presents a recent insight into the management of one of the most common and biggest clinical challenges for a clinicianin young permanent dentitions. The minor oral developmental anomalies present with a mirage of clinical problems with no definite etiology. So a thorough knowledge, complete diagnosis and carefully planned treatment are the key to success for efficiently managing such cases. Besides this, the age of the patient, occlusal status and psychology play an important role as well.

\section{REFERENCES}

1. Jelka Jukie, lija Ckrinjarie, Domagoj Glavina. The Prevalence of Oral and Dental Anomalies in Children with Developmental Disturbances. Acta Stomat Croat 2002;36:79-83.
2. Mitchell L. Supernumerary teeth. Dent Update 1989;16:65-69.

3. Debjit Ray, B Bhattacharya, S Sarkar, G Das. Erupted maxillary conical mesiodens in deciduous dentition in a Bengali girl - A case report. $J$ of Ind Soc Ped and Prev Dent 2005;23(3):153-55.

4. Kavita Mathu-Muju, J Timothy Wright. Diagnosis and treatment of molar incisor hypomineralization. Compend Contin Educ Dent 2007;27:604-10.

5. Weerheijm KL, Jalevik B, Alaluusua S. Molar-incisor hypomineralization. Caries Res 2001;35:390-91.

6. SuhaTurkaslana, Hasan Suat Gokceb, Mehmet Dalkýzc. Esthetic Rehabilitation of BilateralGeminated Teeth: A Case Report. Europ Jour of Dent 2007;1:18891.

7. Blank BS, Ogg RR, Levy AR. A fused central incisor. Periodontal considerations in comprehensive treatment. J Periodontol 1985;56:21-24.

8. Tulunoglu O, Cankala DU, Ozdemir RC. Talon's cusp: Report of four unusual cases. J Indian Soc Pedod Prev Dent 2007; 25:52-55.

9. Danny R Sawyer, Emmanuel O Taiwo, Adeyemi Mosadomi. Oral anomalies in Nigerian children. Community Dentistry and Oral Epidemiology. Volume 12 Issue 4, Pages 269-273. Published Online: 29 May 2006. 